Écrire

l'histoire

\section{Écrire l'histoire}

Histoire, Littérature, Esthétique

12 | 2013

Présent (2)

\title{
Vite, mettre en scène un génocide
}

The Despoiler, de Reginald Barker (1915)

\section{Marc Vernet}

\section{(2) OpenEdition}

\section{Journals}

Édition électronique

URL : http://journals.openedition.org/elh/339

DOI : $10.4000 /$ elh.339

ISSN : 2492-7457

Éditeur

CNRS Éditions

\section{Édition imprimée}

Date de publication : 15 novembre 2013

Pagination : 65-74

ISBN : 978-2-35698-065-6

ISSN : 1967-7499

Référence électronique

Marc Vernet, «Vite, mettre en scène un génocide », Écrire l'histoire [En ligne], 12 | 2013, mis en ligne le 15 novembre 2016, consulté le 23 septembre 2020. URL : http://journals.openedition.org/elh/339 ;

DOI : https://doi.org/10.4000/elh.339 


\section{Vite, mettre en scène un génocide $^{1}$}

\section{The Despoiler, de Reginald Barker (1915)}

Commençons par ce très beau plan que les spectateurs ont pu découvrir à partir du 15 décembre 1915 à New York:

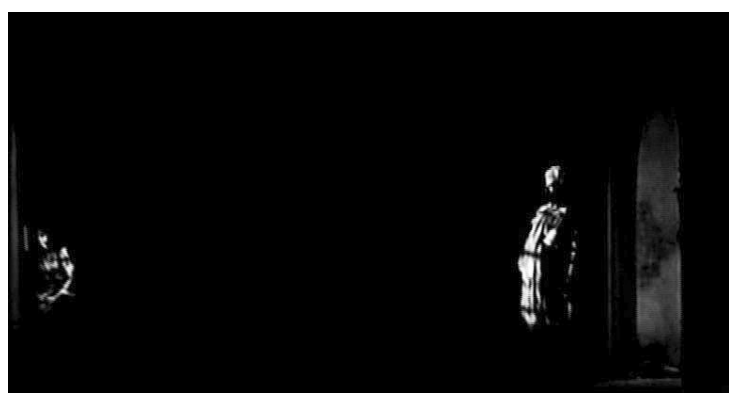

The Despoiler, Reginald Barker (1915). Collection Cinémathèque française. D.R.
Non, on n'y voit pas grand-chose. C'est la nuit, dans une pièce sans lumière. Grâce à une lucarne hors champ à gauche, on distingue deux petites taches lumineuses, toutes deux en bas du cadre, l'une à gauche étant une jeune femme qui va se faire violer, l'autre à droite étant le violeur qui va fermer la porte au verrou. Dans la version initiale, la jeune femme, Béatrice, est chrétienne et vierge; l'homme, le khan Ouârdaliah², est Kurde et lubrique. Ainsi est représenté le génocide arménien.

Ce plan est beau parce qu'il est deux fois osé. Osé dans sa forme et osé dans son fond. Dans sa forme parce que si les scènes de nuit en clair-

Marc Vernet, Univ Paris Diderot, Sorbonne Paris Cité, GÉRILAG-Littérature et civilisation du XIX siècle, EA 4410.

1. Lisant ce texte sur épreuves, un lecteur chevronné me fait observer, à très juste titre et je l'en remercie, qu'en 1915 le terme « génocide » n'existait pas. Il a été créé en 1944 par Raphael Lemkin dans son Axis Rule in Occupied Europe. Il s'applique, pour partie et rétroactivement, à l'extermination des Arméniens par les Turcs en 1915, mais cette phrase, que je feins de mettre dans la bouche de la Triangle Film Corporation, n'a pu être prononcée en 1915 ni par les Turcs (qui refusent encore le terme) ni par les Américains (qui désignaient alors les faits, comme on le verra en son temps, par le terme de « race extermination »). Il s'agit donc d'un énoncé de 2012 sur un film de 1915.

2. Le nom et le titre sont évidemment fictifs. En turc, khan signifie « dirigeant », « celui qui commande ».

Marc Vernet, « Vite, mettre en scène un génocide. The Despoiler, de Reginald Barker (1915) »

Écrire l'histoire, $\mathrm{n}^{\circ} 12$ - automne 2013, 65-74 
obscur sont déjà fréquentes depuis quelques années aux États-Unis, cette composition est tout à fait inédite, par son cadrage, très large et élevé (d'où l'écrasement de la pauvre Béatrice), et dans son fond (mais on a déjà compris que les deux sont indissociables), puisqu'il s'agit de figurer le viol d'une vierge chrétienne par un musulman froidement sadique. L'audace tient au cadrage, à la composition à la fois déroutante (par rapport à un état standard du cinéma de l'époque) et adéquate (par rapport au contenu du film). Elle tient aussi à quelque chose que nous avons perdu en chemin: le teintage du film, qui devait être ici bleu pour les deux personnages afin de représenter la lumière de la Lune pénétrant par la lucarne ${ }^{3}$.

\section{Un film très ambitieux}

L'ambition de The Despoiler se déploie sur plusieurs plans. Il s'agit d'un film Kay Bee, c'est-à-dire produit par la New York Motion Picture Company sous la responsabilité de Thomas H. Ince qui a, pour l'occasion, réuni les plus grands talents de son studio. À commencer par le scénariste, John G. Hawks, un de ses hommes de confiance dans la construction des histoires. Il en va de même pour le réalisateur, Reginald Barker, qui est le bras droit d'Ince, celui à qui il confie les projets les plus stratégiques. En ce qui concerne les acteurs, on a pris les deux plus grands, Enid
Markey pour le personnage féminin et Frank Keenan pour le personnage masculin. Le budget du film (54 160 dollars, dont 10000 pour Keenan) est important pour l'époque: on a privilégié le tournage en extérieurs réels chaque fois que cela était possible pour l'ampleur de l'espace et la véracité des images, les nombreux décors sont des plus soignés, et enfin les figurants sont en nombre, tant du côté des chrétiens que des musulmans, des hommes que des femmes. Un grand soin a été apporté à la mise en scène des groupes, qu'il s'agisse de soldats en action ou de femmes en prière, en privilégiant des chorégraphies de mouvements d'ensemble coordonnés. Cela est confirmé par la durée du tournage, qui s'étend de la mi-août 1915 à début octobre, soit presque deux mois, ce qui est pour l'époque tout à fait exceptionnel.

Le film s'inscrit donc pleinement dans la politique ambitieuse de la Triangle, que l'unité de Thomas H. Ince a officiellement rejointe à sa création, le 20 juillet de cette même année 1915. Il s'agit de fournir chaque semaine aux exploitants de salle deux programmes, chacun constitué d'un long-métrage dramatique et d'un court-métrage comique. La production des films dramatiques est supervisée par David W. Griffith (pour le label Fine Arts) et Thomas H. Ince (pour le label Kay Bee), celle des films comiques par Mack Sennett (pour le label Keystone). L'ambition de la Triangle est d'offrir une production régulière (une fois

3. Je n'ai pas le loisir ici de développer ce que ce teintage en bleu a d'audacieux par rapport au code des couleurs de l'époque. 
par semaine), de qualité (d'où l'importance des superviseurs comme Ince), sur la base d'un longmétrage $^{4}$, avec pour visée la différenciation du programme Triangle et l'augmentation à ce titre du prix des places, de façon à pouvoir accroître encore et en retour les budgets de production. Une des histoires à laquelle le film appartient est donc celle de la production cinématographique américaine au moment de la Première Guerre mondiale: la Triangle tente, à la suite de la $\mathrm{Mutual}^{5}$, une intégration horizontale (trois unités spécialisées de production pour une diversité de produits à qualité constante) et verticale (organisation allant de l'achat de matériel littéraire à l'exploitation en salles). Cette tentative n'est pas seulement «naturelle » (évolution du système de production américain), elle est aussi géopolitique: il s'agit d'organiser au plan national la production pour lui donner une diffusion internationale au moment où le premier producteur mondial, l'Europe, est entré en guerre.

The Despoiler est donc mis en chantier dans ce contexte, et très tôt puisque la nouvelle qui sert de base au film est déposée au nom de ses auteurs, Hawks et Ince, le 11 août à la Library of Congress, afin de figurer parmi les premiers films distribués par la Triangle. Celle-ci ouvre son programme public le 7 novembre et The Despoiler, distribué dans l'État de New York le 15 décembre, prend donc le sixième rang dans l'ouverture de cette nouvelle politique de production et de distribution. Nul doute qu'Ince entend y tenir son rang et donner toute sa place dans l'entreprise Triangle à son unité de production.

\section{La leçon des deux versions}

La seule copie survivante du film connue à ce jour est celle qui est conservée par la Cinémathèque française et qui a fait l'objet, dans le cadre du programme de recherche ANR Cinémarchives, d'une restauration ${ }^{6}$. La version française est distribuée en mai 1917, soit un an et demi après la première sortie américaine, sous le titre Châtiment, et elle témoigne elle aussi d'une certaine audace. L'histoire est assez claire: à la frontière turcoarménienne $^{7}$, les édiles chrétiens d'un bourg,

4. En 1915, les programmes de cinéma sont encore largement constitués de courts-métrages variés, que l'on change au cas par cas en fonction de la réponse du public. L'ambition de la Triangle est d'imposer le long-métrage et le changement total de programme chaque semaine, de façon à réguler en amont la production et à optimiser les profits.

5. Société de distribution et de production indépendante, fondée en mars 1912 par John R. Freuler, Charles J. Hite et Harry E. Aitken, qui la préside jusqu'en mai 1915, date à laquelle il est débarqué par Freuler. C'est alors qu'il crée la Triangle.

6. Voir sur le site Cinémarchives de la Cinémathèque française, <www.cinematheque.fr/sites-documentaires/triangle/rubrique/archivesphotos-et-films-les-films-triangle-la-restauration-de-the-despoiler.php>, cons. 27 févr. 2013. Pour ce qui suit, et pour les dates et faits, je m’appuie fortement sur le travail mené par Loïc Arteaga et Clarisse Bronchti et les éléments qu'ils ont mis au jour. Pour l'interprétation à partir de ces dates et faits, j'en assume seul la responsabilité.

7. Cette frontière est fictive, comme le nom des personnages ou des lieux.

Marc Vernet, « Vite, mettre en scène un génocide. The Despoiler, de Reginald Barker (1915) »

Écrire l'histoire, $\mathrm{n}^{\circ} 12$ - automne 2013, 65-74 
Kérouassi, refusent de répondre aux réquisitions de l'envahisseur kurde, conseillé par un colonel des Empires centraux. Le khan Ouârdaliah impose un ultimatum : si les édiles ne cèdent pas, il fera prisonnières les femmes et les filles qui se sont réfugiées dans une abbaye proche et elles seront déportées par le colonel. Mais Béatrice, la fille unique bien-aimée du colonel, a elle aussi trouvé refuge dans l'abbaye. Ouârdaliah, qui l'a déjà rencontrée et remarquée, l'y découvre, menace de livrer l'abbaye à ses soldats avides si elle ne cède pas à ses avances. La pauvre cède pour sauver ses consœurs ${ }^{8}$, mais elle l'abat après le martyre. Le colonel, arrivé sur place, demande à ce que le coupable lui soit livré. Béatrice paraît entièrement voilée. Ne la reconnaissant pas, il l'abat, découvre son horrible méprise et, accablé, poursuit avec les troupes d'Ouârdaliah leur sinistre chevauchée.

On ne peut être que saisi, dans ce film produit à l'été 1915, par l'actualité du propos, alors que les premiers événéments marquant les génocides arménien et assyrien datent de la fin février 1915. Même implication des Empires centraux aux côtés des Turcs et des Kurdes (ce sont ces derniers qui conduisent le génocide assyrien) dans le sudest de l'actuelle Turquie, mêmes accusations de collusion d'une ville avec l'ennemi ${ }^{9}$, même conflit entre musulmans et chrétiens: le cinéma dénonce par l'image et par les situations, dans une actualité internationale brûlante. Que cela se produise dans le cadre de la politique de la Triangle n'est pas pour étonner: Harry E. Aitken, patron de celle-ci, est celui qui, à la tête de la Mutual, avait réussi à prendre sous contrat Pancho Villa pour pouvoir filmer ses exploits révolutionnaires au Mexique ${ }^{10}$. Il est vrai aussi que les États-Unis - qui en 1915 célèbrent le cinquantenaire de la fin de la guerre de Sécession restent très sensibles à ces questions de guerre civile, au point d'en faire un genre cinématographique sous le nom de « Civil War». Ince, producteur de The Despoiler, en est un des spécialistes, mais son partenaire à la Triangle, Griffith, a démontré en 1915 avec Birth of a Nation qui en était le grand mâ̂tre. On peut encore ajouter ceci: The Despoiler est distribué en France en avril 1917, quand les ÉtatsUnis entrent en guerre, et c'est d'ailleurs aux mêmes dates que le film est aussi ressorti une première fois aux États-Unis ${ }^{11}$. La cohérence et la force de la

8. Mais aussi son père, qui est le supérieur hiérarchique du khan, et les édiles qui résistent au village.

9. La bourgade assaillie par les Kurdes, Kérouassi, fait penser à la résistance des municipalités chrétiennes du Tur Aldin en février 1915 dans le génocide assyrien, et de Van en avril 1915 dans le génocide arménien.

10. Voir Margarita de Orellana, Filming Pancho. How Hollywood Shaped the Mexican Revolution, trad. de l'espagnol par John King, Londres, Verso, 2009, p. 43. On sait que les scènes filmées étaient des reconstitutions jouées, le filmage en direct des actions militaires étant impossible.

11. Il le sera à nouveau en 1920. La levée de la censure américaine commence fin 1916, en plein preparedness, lorsque les villes de San Francisco et de Los Angeles déclarent le film, sous son nouveau titre de War's Women, exploitable nonobstant les précédentes interdictions: «War's Women Wins Against Censorship », Motion Picture World, vol.30, n 7 (18 nov. 1916), p. 990. 
politique de la Triangle s'imposent à travers ce film ambitieux, à la hauteur des enjeux.

Comme l'ont remarqué les restaurateurs de la version française, on note toutefois d'assez nombreuses différences avec la version américaine, pour laquelle on dispose d'un synopsis et d'un scénario $^{12}$ : diffusée dans la seconde moitié du mois de décembre 1915, elle fait preuve d'une grande imprécision quant aux faits évoqués. Il n’y a pas que des différences de métrage entre la copie distribuée en 1915 et celle qui est distribuée en France ${ }^{13}$ en 1917: il y a de grands changements dans les noms des personnages, le lieu de l'action et l'intrigue et sa résolution. Alors que dans la version française le colonel et sa fille se nomment von Werfel, dans la version américaine ils s'appelaient Damien. La ville attaquée n'est pas Kérouassi mais Tournaisse. On n'était pas à la frontière turco-arménienne mais quelque part dans les Balkans (le pays s'appelle Balkania). Dans la version américaine, Damien/Werfel s'assoupissait devant une cheminée et rêvait tout l'assaut de l'abbaye et ses funestes suites: heureux de n'avoir fait qu'un cauchemar, il repartait avec sa fille et ses troupes en épargnant la localité et ses responsables. C'est donc bien la version française qui en 1917 pointe en direction des génocides arménien et assyrien sur la base du matériau américain de 1915, alors que, si l'on en juge par le scénario, la version de 1915 était attentive à brouiller les pistes (noms français, Balkans, fin morale soulageante). Il ne faut donc pas que ce que nous savons d'Ince, de la Triangle et de la version française nous fasse commettre une erreur d'interprétation de la version initiale. Celle-ci est soumise aux conditions de l'époque: les États-Unis sont un pays neutre, qui n'est pas entré en guerre et qui doit donc respecter les belligérants sans s'immiscer dans le conflit. On ne fait référence ni aux Allemands, ni aux Turcs, ni au génocide, et l'on prend soin dans un avantpropos du scénario de préciser qu'il s'agit d'une œuvre neutre, dans laquelle aucun pays et aucune nation ne peuvent se reconnaître, se déroulant en un lieu imaginaire qu'il serait inutile de vouloir identifier et qui a pour seul but de s'élever contre le sort fait aux femmes dans les conflits (d'où le titre War's Women donné au film à sa ressortie, quand les archives indiquent The Despoiler ou The Despoilers pour la sortie). Ce n'est donc pas une intervention politique, mais bien un rappel moral pour que les civils, et au premier rang les femmes, soient épargnés. On peut toutefois noter, dans cette ligne, que le scénario est parfois plus direct et plus cru que le film: dans le scénario, Damien

12. Tous deux conservés à la Cinémathèque française: fonds Aitken-Triangle, boîte 83 cote 1749 au titre de film War's Women. On ne dispose pas du découpage technique, qui aurait montré le texte des cartons, ni de documents pour la version distribuée en 1917 aux États-Unis.

13. Le métrage de la copie conservée est encore plus court que celui de la copie distribuée en France, ce qui ne rend pas plus facile l'interprétation de la copie conservée.

Marc Vernet, « Vite, mettre en scène un génocide. The Despoiler, de Reginald Barker (1915) »

Écrire l'histoire, $\mathrm{n}^{\circ} 12$ - automne 2013, 65-74 
demande à l'émir de s'emparer de l'abbaye et de la mettre à sac, alors que la version française se limite à un ordre de capture à des fins de transport. Ou encore, le scénario montre l'émir en train de blasphémer devant un crucifix et de tenter de briser un verre de vin sur la tête du Christ, alors que cette scène a disparu dans la version française ${ }^{14}$.

À ce point, il semble logique de conclure que la version américaine de 1915 n’avait pas la visée politique que lui a donnée la version française de 1917, en raison du changement de date et de lieu.

\section{Première hypothèse}

Nous avons le scénario américain, et nous avons les images (américaines) et les cartons (français) de la version française. Les images sont tout à fait fidèles au scénario, qui, dans sa version développée ${ }^{15}$, détaille avec précision ce que nous pouvons voir à l'écran en matière d'action. À ceci près toutefois que la caractérisation vestimentaire des personnages contredit largement la prudence des dénominations scénaristiques. Le costume arboré par Damien/Werfel est nettement allemand, même s'il ne comporte pas d'insigne explicitement allemand (en tout cas il n'est en rien français, comme pourrait le laisser entendre le patronyme). Quant à celui de l'émir/khan, il est, dans sa flamboyance, très turc, ou ottoman, et très peu balkanique (imposant turban, étoffes chamarrées, sabre...). Linguistiquement, le film est prudent, mais visuellement il est beaucoup plus indicatif, même si les costumes n'affichent pas leur nationalité. Un indice doit retenir notre attention: le titre du scénario a été déposé le 11 août 1915, et le texte du scénario le 31 (et le tournage commence le 20 pour se terminer le 20 octobre). Le film est exploité à partir du 15 décembre, le titre est déposé le 19 décembre ${ }^{16}$. Nous sommes toujours là dans la ligne de la politique d'Ince et de la Triangle, selon une procédure réglée et rodée: acquisition des droits sur le scénario enregistré à Washington, enregistrement du titre du film puis dépôt de la copie à Washington également, une fois le film passé devant le National Board of Censorship de New York. Nous sommes toujours là dans la politique régulière : le film, tourné sur la côte Ouest, est expédié pour tirage sur la côte Est où il passe devant la commission de contrôle qui indique éventuellement ce qui doit être censuré ainsi que le nombre de copies qui peuvent être tirées pour l'exploitation. The Despoiler a suivi la procédure à la lettre et

14. Comme c'était sur cette scène que portait la censure de 1916, il se peut tout à fait que le remontage constaté dans la version française de 1917 ait été celui de la version américaine de 1917.

15. Le fonds français comporte également un synopsis, beaucoup plus court, très édulcoré par rapport au scénario, sans doute destiné, après tournage et montage, à la presse et aux distributeurs.

16. Notons que la copie du film n'est enregistrée que le 16 janvier 1918, alors qu'au 19 décembre 1915 on n'enregistre qu'un photoplay, c'est-à-dire sans doute le découpage technique du film correspondant à la copie exploitée. 
en a franchi les différentes étapes: la commission nationale de censure n'a rien trouvé à redire au film qui peut être exploité tel quel au sein du tout nouveau programme Triangle. Ce passage devant la commission de censure est voulu par les producteurs: cela les assure (ou devrait les assurer) d'une exploitation nationale sans encombre et donc sans retard dans l'exploitation et sans frais dans d'éventuels ajustements tardifs.

Mais il se trouve que le film est, par les responsables de la Triangle, retiré de l'exploitation le 20 janvier 1916, à la suite d'une première plainte fin décembre - pour indécence - de la part d'un membre de la commission de censure municipale (et non pas nationale) de New York. Ce monsieur, George H. Bell, s'offusque de l'indécence de certaines scènes (celles par lesquelles nous avons commencé) et notamment de l'agression de la figure du Christ en croix par l'émir. Ce sont les producteurs qui retirent le film, c'est un individu, membre d'une organisation municipale, qui se substitue à un organe, collectif et anonyme, réputé d'ampleur nationale. Il y a deux choses qui ne vont pas: un individu intervient après le passage du film devant la commission de censure qui l'autorise, le film n'est pas remonté (en supprimant les scènes incriminées, ce qui en l'occurrence était faisable comme en atteste la version française où l'attentat au crucifix ne figure pas), mais tout simplement retiré de la cir- culation. Geste étonnant quand on sait le soin mis à sa réalisation et l'ampleur du budget qui y a été consacré. De plus, début 1916, la Triangle connaît ses premières difficultés financières et ce n'est sûrement pas le bon moment pour arrêter l'exploitation d'un film coûteux et talentueux. Ma première hypothèse est la suivante: le censeur municipal est un homme de paille et son propos, détourné. Ce qui est visé n'est pas l'atteinte à la religion ou l'indécence des vêtements déchirés de Béatrice, mais bien la situation en Turquie et le rôle qu'y joue l'Allemagne. Autrement dit, le censeur n'a pas cru la lettre : il a cru l'image, et il masque par une censure religieuse une censure commandée de nature politique. Les États-Unis étant neutres, et la production cinématographique américaine très occupée par le conflit en Europe et au Mexique, la délégation allemande aux États-Unis est extrêmement vigilante à ce qui est représenté dans les films et elle ne manque pas de faire savoir quand elle estime les règles de la neutralité outrepassées et les références à l'Allemagne trop transparentes.

Cela est confirmé par ce qui se passe à Chicago dans la première semaine de janvier: le bureau de censure de la ville interdit totalement le film, qui devait être présenté conjointement à Don Quixote et The Hunt au Studebaker ${ }^{17}$ : cette salle ne pourra présenter qu'un programme amputé, aucun visa n'ayant été accordé par la censure pour The Des-

17. Le Studebaker est une salle de prestige, louée par la Triangle au même titre que le Knickerbocker Theater de New York, où les programmes Triangle sont présentés en exclusivité pour inciter ensuite les autres exploitants à les louer.

Marc Vernet, « Vite, mettre en scène un génocide. The Despoiler, de Reginald Barker (1915) »

Écrire l'histoire, $\mathrm{n}^{\circ} 12$ - automne 2013, 65-74 
poiler ${ }^{18}$. Il s'agit bien d'une censure totale, valant pour le film dans son entier et non pour telle ou telle scène, et si elle frappe si durement, c'est sans doute parce que la région a accueilli une très importante communauté allemande qu'il s'agit de ne pas heurter. L'appel à censure à New York était arrivé trop tard (le visa avait déjà été accordé), mais à Chicago le couperet tombe avant toute exploitation. On peut comprendre que, dans ces conditions, la Triangle préfêre, le 20 janvier, retirer le film du circuit et rebâtir son programme hebdomadaire avec un autre titre pour ne plus encourir dans d'autres territoires les mêmes risques d'amputation de la programmation.

\section{Seconde hypothèse}

Mais le cinéma américain avait-il les moyens de savoir et de représenter ce qui se passait depuis février et avril 1915 au sud-est de la Turquie actuelle? Les Turcs, dès le départ, ont refusé de reconnaître les massacres comme leur responsabilité, et ont œuvré partout pour en nier l'existence. Il nous faut faire un nouveau virage sur l'aile. La Triangle, et notamment Griffith, est très proche de l'administration Wilson, celui-là même qui veut maintenir les États-Unis à l'écart du conflit tout en sachant que ce sera très difficile. Février 1915: début du génocide en Arménie. Avril 1915: torpillage du Lusitania. Mai 1915: premiers mouve- ments à la Mutual pour la création de la Triangle, dont le premier programme, à l'automne 1915, comporte plusieurs films traitant de conflits: The Coward - supervisé par Ince - pour la guerre de Sécession, The Lamb - supervisé par Griffith - pour les troubles à la frontière américano-mexicaine, et même A Submarine Pirate - supervisé par Sennett pour la lutte contre les sous-marins. Il ne serait pas anormal que The Despoiler vienne s'inscrire dans ce cadre, dans cette visée à la fois concernée et pacifiste: les États-Unis regrettent l'état de guerre, veulent la paix mais ont les moyens de se défendre (en cas d'invasion). Sauf que les mauvaises nouvelles continuent d'arriver et qu'il est difficile de résister en maintenant une stricte neutralité.

Parmi les mauvaises nouvelles, il y a les dépêches de Henry Morgenthau senior, autre proche de Woodrow Wilson. Il n'a pas pu faire partie de son gouvernement, mais depuis 1913 (et jusqu'en 1916), il est pour l'administration Wilson ambassadeur des États-Unis à Constantinople. Son rôle, sa morale, ses origines juives le rendent particulièrement sensible aux jeux politiques qui conduisent aux massacres en masse de chrétiens en territoire musulman avec l'aide des Allemands. Dès décembre 1914, il alerte à propos de l'agression par les Turcs d'une institution d'enseignement chrétienne à Constantinople,

18. «The Gurrent Triangle Bill, reviewed by Neil G. Caward », Motography, vol. 15, no 2 (8 janv. 1916), p. 84. La revue, très anticensure, se moque des censeurs et raconte le film sur la base des commentaires élogieux publiés à New York, puisque leur journaliste n’a pu voir le film censuré. Cette place donnée au résumé est justifiée par le fait que cela peut intéresser les exploitants qui, dans une autre ville ou dans un autre État, pourraient louer le film. 
Notre-Dame de Sion, ce qui n'est pas sans nous rappeler l'abbaye où Béatrice trouve la mort par la faute du khan. Il signale très tôt les événements de Constantinople (rafle et déportation de 600 notables arméniens) et ceux de Van. Son langage est peu diplomatique: dans une dépêche en date du 15 juillet 1915, où il demande instamment si on a bien reçu et bien tenu compte de la précédente dont il reste sans nouvelles, il rappelle qu'on est en face d'un véritable génocide ${ }^{19}$ ("race extermination ", selon ses propres termes) des chrétiens d'Arménie.

Les dépêches de Morgenthau senior ne seront rendues publiques qu'en 1918 avec la publication de ses Mémoires sous le titre Ambassador Morgenthau's Story (New York, Doubleday) et l'année suivante en France chez Payot. Mais ma seconde hypothèse est là: l'administration Wilson a tenté, notamment via la Triangle, de traiter par le cinéma de fiction ce qu'elle ne voulait pas entreprendre par l'action politique, dans le triple but d'informer le public, de maintenir sa position pacifiste, mais aussi de préparer l'éventuelle entrée en guerre, ce que recouvre bien le terme de "preparedness » utilisé alors pour définir l'action psychologique des États-Unis.
La leçon de The Despoiler, en partie fondée sur l'épisode biblique de Judith de Béthulie ${ }^{20}$, est que les parents (pacifistes ou non) doivent se préparer à voir leurs enfants se sacrifier pour défendre les valeurs fondamentales. L'assaut de l'abbaye est la métaphore du viol immontrable de Béatrice, lui-même métaphorisé par une prise de vin forcée, viol qui vaut ici à la fois pour l'agression guerrière et pour les génocides arménien et assyrien, lesquels sont à leur tour à prendre comme la métaphore de la Première Guerre mondiale. Damien et Tournaisse, les noms donnés dans la version américaine, ne sont pas là tout à fait par hasard: l'invasion de la Belgique par les troupes allemandes a provoqué un tollé aux États-Unis, les pires horreurs étant racontées sur le comportement des soldats allemands vis-à-vis de ce petit pays neutre et de ses civils ${ }^{21}$. Chaque signe de l'agressivité allemande met à mal la position de Wilson, chaque représentation américaine de l'agressivité allemande met sur les nerfs l'ambassade allemande. Ce sont dans ces contraintes qu'est né The Despoiler pour vivre sa très courte puis relancée carrière. Il est toutefois peu probable que le distributeur français de Châtiment ait eu

19. Aujourd'hui encore la Turquie refuse le terme et parle pour les événements de 1915-1916 de «prétendu génocide arménien ».

20. Pour mémoire: Judith, déçue par l'attitude attentiste de son peuple face au tyran Holopherne qui assiège Béthulie, décide d'agir seule. Elle va nuitamment retrouver le tyran sous sa tente, le charme, le fatigue et le soûle pour ensuite lui couper la tête et la ramener (dans un sac) aux siens. Elle impose ainsi la paix. Béatrice, dans son martyre, entend épargner les femmes, les édiles, son père et la ville de Kérouassi. Rappelons qu'en 1914 Griffith a réalisé une Fudith of Bethulia pour la Biograph, dont c'était le premier long-métrage.

21. Sur les atrocités à l'égard des civils, voir John Horne, « Atrocités et exactions contre les civils », dans Stéphane Audoin-Rouzeau, JeanJacques Becker (dir.), Encyclopédie de la Grande Guerre, Bayard, 2004, p. 367-380.

Marc Vernet, « Vite, mettre en scène un génocide. The Despoiler, de Reginald Barker (1915) »

Écrire l'histoire, $\mathrm{n}^{\circ} 12$ - automne 2013, 65-74 
la possibilité de retravailler le matériau de 1915 pour l'actualiser: il est beaucoup plus probable que la version française de 1917 découle en fait de la version américaine de 1917, après le retrait de janvier 1916 et au moment de l'entrée en guerre des États-Unis, en avril 1917. Au fond, la version de 1917, américaine et française, ne fait que gommer un peu la censure auto-imposée de 1915 (flou des localisations, des appellations et du procédé du personnage qui n'a fait que rêver) tout en maintenant la censure imposée (la scène jugée blasphématoire). 